\title{
Propuesta de un enfoque dinámico grupal para el tratamiento combinado de los trastornos esquizofrénicos.
}

\author{
Group psychotherapeutic approach in schizophrenia.
}

\author{
Margarita Silvestre García a , Joaquín Ingelmo Fernández ${ }^{\text {b }}$, Alfonso García-Ordás Álvarez ${ }^{\text {c }}$ \\ ${ }^{a}$ Facultad de Medicina de la Universidad de Extremadura, España. ${ }^{b}$ Complejo Hospitalario Universitario \\ de Badajoz, España. ${ }^{c}$ Equipo de Salud Mental de Valdepasillas, Badajoz, España.
}

Correspondencia: Margarita Silvestre García (marsilve@telefonica.net)

Recibido: 10/07/2012; aceptado: 01/12/2012

\begin{abstract}
RESUMEN: El presente trabajo trata de reflexionar teóricamente sobre una experiencia grupal, realizada durante varios años con pacientes esquizofrénicos. Lógicamente, siempre en el contexto de un tratamiento combinado de los pacientes (psicofármacos, psicoterapia, terapias rehabilitadoras). La creación de un espacio terapéutico grupal para el tratamiento de estos pacientes diagnosticados de esquizofrenia lleva implícita la idea de que estos pacientes puedan reunirse para poder estar, articular e integrar las experiencias de forma compartida, tanto las inherentes a la propia patología esquizofrénica, como las procedentes de los diferentes contextos terapéuticos en los que son atendidos y, obviamente, las experiencias vitales de cada cual. En este sentido, la psicoterapia grupal constituiría el elemento básico y el soporte de las experiencias que estos pacientes realizan a lo largo de su proceso de enfermedad.

PALABRAS CLAVE: Psicoterapia de grupo de orientación dinámica. Esquizofrenia. Tratamientos combinados. Psicofármacos. Terapias rehabilitadoras.
\end{abstract}

\begin{abstract}
The present study attempts to reflect theoretically on a group experience conducted over several years with schizophrenic patients, in the logical context of combined treatment (psychiatric drugs, psychotherapy, rehabilitation therapies). The creation of a therapeutic group space for the treatment of patients diagnosed with schizophrenia carries with it the implicit notion that these patients meet to articulate and integrate in a shared way their experiences as schizophrenic patients, their experiences resulting from the various kinds of therapeutic attention, and obviously their individual life experiences. In this sense group psychotherapy constitutes the basic element and support of the experiences that these patients encounter during the process of their illness.

KEY WORDS: Dynamic-oriented group therapy. Schizophrenia. Combined treatments. Psychiatric drugs. Rehabilitation therapies.
\end{abstract}

\section{Introducción}

En la actualidad la esquizofrenia se considera como una enfermedad de etiología multifactorial, que incluye, por una parte, alteraciones neurobiológicas que determinan anomalías en el funcionamiento psicológico (nivel clínico: los síntomas diagnósticos del trastorno) y, por otra, una vulnerabilidad reactiva a factores externos, que están también implicados como factores causales, manifestándose como formas de interacción, tanto consigo mismo como con el entorno interpersonal (nivel dinámico). La esquizofrenia es, por tanto, una enfermedad en la que los facto- 
res biológicos, psicológicos y ambientales confluyen para dar lugar a un trastorno heterogéneo, que cursa como un proceso, con múltiples fases (aguda, residual, en remisión) y que presenta manifestaciones psicopatológicas de curso crónico. Las alteraciones del pensamiento y de los procesos senso-perceptivos, interfieren en la propia percepción y en la del entorno. Estas alteraciones, mantenidas en el tiempo, determinan un deterioro en las capacidades de auto-cuidado y autonomía, en el establecimiento y mantenimiento de relaciones familiares y sociales saludables y dificultades para desarrollar actividades laborales y lúdicas. De esta manera, se crean relaciones de dependencia emocional intensa y mutua entre el paciente y los otros significativos de su entorno interpersonal (1). En términos generales, y en la mayoría de los casos, estos pacientes dependen durante gran parte de su vida de su familia y del sistema público de salud.

Al plantear estrategias terapéuticas para abordar la esquizofrenia, hay que tener en cuenta las premisas etiológicas y evolutivas que hemos señalado. El abordaje terapéutico de estos pacientes debería estar basado en la integración de todos los aspectos (biológicos, psicológicos, familiares, sociales) que están implicados en la producción de esta patología.

Plantear hoy día tratamientos excluyentes (fármacos o psicoterapia) en la esquizofrenia supone olvidar todo una saber acerca de esta forma de enfermar e imponer una limitación en las posibilidades de salud y bienestar de estos pacientes. En este sentido, los tratamientos combinados (psicofármacos, psicoterapia y técnicas de rehabilitación) constituyen, hoy día, la indicación terapéutica específica en el abordaje terapéutico de la esquizofrenia.

Las reflexiones que pretendemos hacer parten de estas conceptualizaciones sobre la esquizofrenia y, como hemos señalado anteriormente, son el resultado de una experiencia de varios años de duración en el tratamiento de este tipo de pacientes, en el contexto de un espacio grupal. Un espacio grupal en el que se integren los conocimientos actuales sobre la etiología (biológicos/psicodinámicos), sobre el desarrollo temporal de esta patología (fases aguda, remisión, residual), sobre las dependencias mutuas que establecen con el mundo externo (familiar y socio-sanitario) y todos los tipos de intervenciones terapéuticas que estos pacientes reciben a lo largo de su vida, en los distintos dispositivos asistenciales (equipos de salud, hospitales, centros de rehabilitación, etc.). La creación de este espacio terapéutico grupal de orientación dinámica lleva implícita la idea de que los pacientes diagnosticados de esquizofrenia, pueden reunirse para poder estar, articular e integrar sus experiencias de forma compartida, tanto las inherentes a su patología, como a las diferentes intervenciones terapéuticas que realizan en los diferentes contextos terapéuticos en los que son atendidos. De esta manera, la psicoterapia grupal constituye el elemento básico y el soporte del resto de las intervenciones terapéuticas que estos pacientes realizan a lo largo del su proceso de enfermedad. 
ORIGINALES Y REVISIONES

Los tratamientos psicoterapéuticos en pacientes graves no se suelen realizar de forma sistemática en nuestro sistema de atención y creíamos que algunos de estos pacientes se podrían beneficiar de este tipo de intervenciones.

La elección del espacio grupal, vino determinada por las siguientes razones. En primer lugar, porque el cambio psicoterapéutico es un proceso complejo que sucede a través de un intrincado intercambio de experiencias humanas y el espacio grupal constituye un contexto privilegiado para el establecimiento de relaciones interpersonales, siendo un lugar, donde los pacientes pueden tener un encuentro con otras personas en situación similar a la suya, con el fin de reflexionar y vivenciar nuevas pautas de interacción más adaptadas a la realidad. El espacio grupal posibilita un encuentro humano, no sólo con el terapeuta, sino, y sobre todo, con el resto de los compañeros del grupo, donde poder expresar y cambiar procesos relacionales patológicos desadaptados. En segundo lugar, porque el espacio grupal es un espacio integrador donde es posible, por una parte, trabajar los aspectos psicológicos de esta patología y, por otra, elaborar e integrar las experiencias que el paciente tiene en el resto de los espacios terapéuticos (tratamientos farmacológicos, ingresos hospitalarios en fases agudas, intervenciones familiares, tratamientos rehabilitadores). Y, por último, el grupo no es solo un espacio integrador de los diferentes niveles de intervención para los pacientes, sino que también para los profesionales que atienden a esos pacientes: el espacio grupal permite valorar las necesidades de atención en cada momento concreto e indicar las intervenciones terapéuticas a seguir en cada caso.

El tratamiento psicoterapéutico de la esquizofrenia, como el de cualquier otro trastorno mental grave, debe asentar sobre tres pilares fundamentales (2). En primer lugar, sobre la denominada teoría del trastorno o teoría de la enfermedad, que debería responder a la pregunta ¿cómo se produce el trastorno?, indicando, obviamente, la especificidad de la producción del mismo. En segundo lugar, sobre la denominada teoría de la cura o teoría del cambio, que debería dar cuenta de los cambios que tendrían que llevarse a cabo para modificar esta patología, teniendo en cuenta, lógicamente, la especificidad del cambio que se pretende realizar. Y, por último, sobre la denominada teoría de la técnica, que trata de poner en claro cuáles son los instrumentos técnicos más adecuados para poder instrumentalizar el cambio específico que se pretende conseguir.

Para poder abordar psicoterapéuticamente a un paciente que padece esquizofrenia es necesario tener en cuenta las teorías que hemos señalado más arriba. En este sentido, lo primero que habría que hacer es establecer cuáles son los elementos específicos que se han puesto en juego para que el paciente desarrolle esta y no otra psicopatología. Por otra parte, habría que plantearse los cambios específicos que queremos producir en los pacientes. Y, por último, establecer cómo hacer esos cambios, mediante qué intervenciones técnicas específicas. Si no tenemos en cuenta 
las diferentes especificidades a las que acabamos de hacer referencia, correríamos el riesgo de crear iatrogenia con intervenciones, que aún siendo correctas desde el punto de vista psicodinámico, podrían ser terapéuticas para un determinado tipo de patología y de estructura de la personalidad e iatrogénicas para otros tipo de patología y de estructura de la personalidad (3). De acuerdo con los planteamientos que acabamos de realizar, cabría reflexionar sobre los tres elementos a los que hemos hecho referencia: teoría del trastorno, teoría de la cura y teoría de la técnica.

\section{Teorías dinámicas sobre las psicosis (teorías del trastorno psicótico)}

Freud, a todo lo largo de su obra, plantea que los pacientes psicóticos no son abordables mediante psicoterapia de orientación analítica (4), al no ser capaces de desarrollar el fenómeno de la transferencia, debido al retraimiento narcisista que surgen en la relación con el objeto. Desde Freud, numerosos autores, plantearon que, atendiendo a la forma particular de relacionarse con los objetos, el abordaje terapéutico de las psicosis debería adecuarse a este tipo de relación, siendo necesarios cambios en la técnica que hicieran posible trabajar las alteraciones relacionales que presentaban (5-7). Técnicas que serían diferentes, lógicamente a las utilizadas en las neurosis $(8,9)$. En términos generales, todos estos autores coinciden en que la problemática básica de las psicosis tiene que ver con las relaciones objetales, con lo vivido en la relación con el otro y en cómo se piensan y se significan estas relaciones o en como no se piensan ni se significan esas relaciones. Este aspecto relacional va a ser, por tanto, el foco de atención de las intervenciones terapéuticas en el tratamiento de la esquizofrenia $(10,11$, entre otros).

Por otra parte, Freud (1911) plantea la existencia de un proceso psicológico que permite que el sujeto diferencie los estímulos de mundo exterior de los estímulos del mundo interior, distinguiendo así entre lo que percibe y lo que se representa. Este proceso diferenciador estaría alterado en la esquizofrenia, confundiendo el sujeto lo que está ocurriendo en la realidad con lo que esta ocurriendo en su interior. El concepto de prueba de realidad (capacidad para diferenciar lo que es percibido de lo que es representado, es decir, la capacidad de diferenciar el mundo exterior del mundo interior) está alterado en la esquizofrenia.

Por último, Freud, desde los inicios de su obra (Freud, 1894), intentó precisar los mecanismos de defensa específicos que estaban implicados en el hecho psicótico. En este sentido, Freud (1927 y 1940, entre otros textos), describió un mecanismo de defensa específico de las psicosis y de algunas perversiones (fetichismo, especialmente): la verleugnung (renegación/desmentida, según la traducción castellana de la obra de Freud que se use). Y, ¿en que consiste, según Freud, la renegación/desmen- 
ORIGINALES Y REVISIONES

tida? Se trata de un mecanismo de defensa mediante el cual el yo intenta deshacerse de una representación intolerable procedente del mundo exterior y sustituirla por una representación inversa: es decir, algo de la realidad exterior que resulta intolerable es rechazado por el sujeto $\mathrm{y}$, por tanto, no entra a formar parte de sus representaciones del mundo y de él mismo, siendo sustituida por una fantasía de contenido inverso. Se trata, por tanto, de un mecanismo que hace posible no dar crédito a un a experiencia externa que resulta intolerable para el sujeto, o, por decirlo en un lenguaje más llano, se trata de un mecanismos que permite "hacer la vista gorda" ante una experiencia traumatizante de la realidad externa (13). Pero, al mismo tiempo, se trata de un mecanismo que, como señala Anna Freud (14), permite sustituir esa realidad inaceptable por una fantasía completamente opuesta a la realidad desagradable. En resumen, diríamos que la psicosis está relacionada con el rechazo de alguna parcela de la realidad exterior mediante un mecanismo específico que consiste en no atribuir significado a la experiencia (negativa a atribuir un sentido a lo percibido), creándose dificultades para poder pensar aspectos de la experiencia habida con la realidad, es decir, en la relación interpersonal con los otros.

Posteriormente, Klein (15) y alguno de sus discípulos (13) entendieron la renegación/desmentida como un mecanismo consistente en rechazar cierta parte de la realidad psíquica porque ésta resultaba intolerable para el sujeto. Es decir, la renegación/desmentida, en términos kleinianos, es un mecanismo que permite no tomar en consideración algo existente en la realidad interior del sujeto y que le resulta insoportable, en "hacer la vista gorda" ante algo de la realidad interna del sujeto: por ejemplo, no tomar en consideración la dependencia respecto de los objetos, dejar de lado los aspectos malos de los objetos con lo que se mantiene la idealización de los objetos, rechazar una determinada imagen de uno mismo, etc.

Sin embargo, la renegación/desmentida no es el único mecanismo de defensa que los kleinianos, al igual que Freud, plantean para dar cuenta de los trastornos psicóticos. Klein en el mismo trabajo (15) describió la identificación proyectiva. Mediante este mecanismo, ampliado posteriormente por otros autores kleinianos, el paciente se libera de las partes del self que le resultan intolerables. Realmente, la renegación/desmentida y la identificación proyectiva son los mecanismos que hacen posible las relaciones narcisistas de objeto, como ya mostró Freud en su trabajo de 1910 sobre Leonardo (16).

Posteriormente, algunos autores kleinianos (19-21) se ocuparon del tratamiento de la esquizofrenia desde modelos de acercamiento diferentes. Por ejemplo, Bion (19) teoriza sobre la incapacidad en la psicosis de dotar de sentido a la experiencia y propone que esta incapacidad se debe a la agresividad del sujeto hacia sí mismo, que ataca su capacidad de dar sentido a las experiencias. Bion (19) insiste que en la psicosis está alterada la capacidad para pensar la experiencia y de dotarla de sentido y significado. 
Por su parte, los autores del denominado grupo independiente británico (22) aportan conocimientos importantes para la compresión de la génesis de las psicosis. Por ejemplo, Winnicott (23) aporta el concepto de sostenimiento y Balint (24) el concepto de falta básica, ambos de gran relevancia para la compresión de las psicosis.

En los últimos tiempos, se han realizado algunos aportes fundamentales para la comprensión de la génesis de las psicosis: el concepto de trastornos por déficit $(10,25)$. Killingmo $(10,11)$, por ejemplo, plantea que en los trastornos por déficit algo de lo que el sujeto ha experienciado/vivenciado no ha sido significado. No ha sido dotado de sentido por el sujeto. Plantea que esta ausencia de sentido provoca un déficit en la estructuración del psiquismo del sujeto, la cual se debería a que el objeto externo no cumplió con la función de dotar de sentido a la experiencia. Por su parte, Bleichmar (3), propone que dentro de la patología por déficit es posible distinguir, cuando menos, tres tipos diferentes de la misma. En primer lugar, la denominada patología por déficit del objeto externo, es decir, una situación en la que el objeto externo dejó de aportar algo esencial para la constitución de cierta función del psiquismo. En segundo lugar, la denominada patología por trauma en la que el objeto externo realmente persiguió, aterrorizó, culpabilizó, traumatizo física y psíquicamente al sujeto. Y, por último, la denominada patología por inscripción patológica originaria, cuando la patología resulta, en lo fundamental, de la identificación del sujeto con la patología de los padres.

En psicoanálisis, clásicamente, se considera que los síntomas presentados por el paciente son el resultado de un conflicto (teoría del conflicto) entre los diferentes sistemas estructurales de la personalidad (yo, ello y superyó). Ahora bien, y como ha señalado reiteradamente Killingmo $(10,11)$ para que se produzca un conflicto es necesario, en primer lugar, que exista un cierto grado de diferenciación entre las diferentes estructuras de la personalidad (yo, ello y superyó): es decir, estas estructuras tienen que estar creadas y diferenciadas. En segunda lugar, tiene que existir una clara diferenciación entre la representación del sí mismo y la representación del objeto. Y, en tercer lugar, el sujeto tiene que haber alcanzado un nivel de desarrollo que le permita utilizar el mecanismo de la represión.

Los síntomas psicóticos no pueden ser explicados mediante el mecanismo de conflicto intrpsíquico, ya que estos pacientes no han alcanzado este nivel de desarrollo y la estructura del yo está sin construir o está gravemente dañada. En las psicosis se dan fallas intrasistémicas (10), como, por ejemplo, una estructura defectuosa del sí mismo, una ausencia de diferenciación entre el yo y el no-yo, fenómenos de difusión de la identidad, falta de capacidad para relacionarse emocionalmente con los objetos (26).

En las psicosis se dan fallas a niveles muy primitivos del desarrollo, no habiendo alcanzado estos sujeto una estructura yoica estable, capaz de interaccionar 
ORIGINALES Y REVISIONES

con el exterior y con el interior del sujeto. Debido a ello, los síntomas presentados por los psicóticos se explican mejor desde la teoría del déficit que desde la teoría del conflicto. Las manifestaciones clínicas (alteraciones del contenido del pensamiento, alteraciones senso-perceptivas, etc.) pueden ser entendidas mejor desde estos enunciados teóricos que desde los enunciados teóricos clásicos del psicoanálisis.

\section{Teoría de la cura en las psicosis}

El cambio que se pretende producir en los sujetos que presentan un cuadro psicótico tiene que ver, fundamentalmente, con la adquisición de unos niveles de desarrollo yoico que les permita salir de las dependencias mutuas en las que están atrapados en sus vínculos con sus objetos significativos y, por tanto, que les permita funcionar con autonomía $(1,3)$. Las intervenciones terapéuticas deben ir encaminadas, por tanto, a este fin de conseguir la autonomía personal y deben estar pensadas y diseñadas para aportar, en la medida de lo posible, los elementos externos de los que estuvo privado en su desarrollo $(3,10,11)$.

Por tanto, la teoría de la cura de las psicosis no va a tratar de hacer consciente lo inconsciente, siguiendo los planteamientos más clásicos del psicoanálisis en los casos de trastornos por conflicto, sino que se va a tratar de dar al paciente aquello que le faltó en sus vínculos con sus objetos significativos. Siguiendo los planteamientos de Killingmo $(10,11)$ no se trata de desvelar ningún significado inconsciente, sino de construir un significado que se quedó sin construir porque el objeto externo no aportó los elementos necesarios para ello; en otros términos, las intervenciones terapéuticas deben ir encaminadas a dar sentido a aquellos acontecimientos vividos por el sujeto que quedaron inscritos sin significado. Planteamientos similares a los que acabamos de señalar, siguiendo básicamente las aportaciones de Killingmo, los podemos encontrar en otras autores psicoanalíticos de orientaciones teóricas muy diferentes: por ejemplo, Aulagnier (17) y Ogden (18), entre otros.

Siguiendo los planteamientos de Killingmo tendríamos que plantear como funciones básicas en el tratamiento de este tipo de pacientes, por una parte, ayudar al paciente a reconocer lo que le ha ocurrido y le ocurre en la relación con el objeto significativo $\mathrm{y}$, por otra, el poder dotar de significado esa experiencia relacional. 


\section{Teoría de la técnica en la psicosis}

Para conseguir los objetivos señalados en el apartado anterior de la teoría de la cura de las psicosis, y siguiendo los planteamientos de Killingmo (11), la técnica psicoterapéutica en las psicosis está centrada en las denominadas intervenciones afirmativas, que pretenden afirmar y validar la experiencia tenida por el paciente en el vínculo con los otros significativos. Con anterioridad a Killingmo, otros autores ya plantearon intervenciones afirmativas: por ejemplo, la contención bioniana (27) y el sostenimiento winnicottiano (23), pueden entenderse como intervenciones de este tipo.

En otros términos, se trata, a través de la intervención del terapeuta, de confirmarle al paciente que lo que percibe y siente es correcto. Lógicamente, las intervenciones afirmativas no son siempre verbales, en ocasiones pueden ser no verbales. En términos generales, podría decirse que en las intervenciones afirmativas se describe la situación que el paciente está pasando y se recogen los sentimientos que se están teniendo. De tal manera que al captar y recoger el terapeuta los sentimientos que el paciente esté sintiendo en esos momentos, los válida y le da al sujeto un reconocimiento sobre su capacidad de sentir y percibir adecuadamente.

\section{Los aportes de las neurociencias}

Los aportes de las neurociencias en relación con los diferentes tipos de memoria son también de gran importancia en la comprensión y abordaje terapéutico de este tipo de patología psicótica. La neurociencia habla, en términos generales, de dos tipos de memoria (28): por una parte, la memoria declarativa, mediante la cual se conservan los recuerdos que pueden ser verbalizados y, por otra, la memoria procedimental que conserva los recuerdos que quedaron inscritos mediante esquemas de acción-emoción, los modos de estar con los otros, etc., que se repiten en las interacciones con los otros.

Numerosos autores han insistido, en los últimos tiempos, siguiendo planteamientos de autores más antiguos (23, por ejemplo), en que los cambios que se producen durante la psicoterapia no están relacionados tan directamente como se creía con las técnicas interpretativas, sino que tienen mucho que ver con todo lo no verbal. Estos cambios terapéuticos tienen que ver con la relación que se establece entre los miembros del grupo y el terapeuta. Las relaciones que se establecen entre los miembros del grupo, incluido el terapeuta, nos van a permitir poner en claro relaciones patológicas y nos van a dar la posibilidad de modificarlas a través de nuevas relaciones, que van a permitir que los pacientes 
ORIGINALES Y REVISIONES

exploren y pongan en acción nuevas formas de estar con los otros más seguras y gratificantes.

Utilización de un formato grupal para las intervenciones psicoterapéuticas en el tratamiento de la esquizofrenia

Cuando nos planteamos iniciar este trabajo con pacientes esquizofrénicos, y teniendo en cuenta las diferentes conceptualizaciones teóricas y técnicas a las que hemos hecho referencia a lo largo del trabajo, consideramos que existían múltiples razones para elegir un formato de psicoterapia grupal de intervención. En otros términos, el grupo psicoterapéutico nos va a permitir realizar actividades que la terapia individual no hace posible. En primer lugar, el grupo es un espacio para la interacción y el intercambio interpersonal, equiparable a cualquier espacio social y familiar en que los seres humanos nos movemos habitualmente, con lo cual existe una similitud entre los espacios terapéuticos grupales y los sociales. En segundo lugar, el grupo por estar constituido por numerosos pacientes (en nuestra experiencia trabajamos siempre con diez pacientes en grupos de apertura lenta) va hacer posible que existan relaciones intertransferenciales múltiples, que aportan un abanico, con sus similitudes y sus diferencias, de experiencias relacionales de las que es posible aprender. En tercer lugar, permite que no se dé una relación terapéutica exclusiva y única con otro miembro del grupo (generalmente, con el terapeuta), que desde lo contratransferencial sobrecargaría mucho emocionalmente al terapeuta y desde el paciente facilita la creación de lazos relacionales cargados (o, mejor, sobrecargados) de ansiedades paranoides y de sentimientos de dependencia, teniendo en cuenta que los pacientes mentales graves tienen tendencia a establecer relaciones de intensa dependencia con los otros. En cuarto lugar, supone un marco idóneo para el encuentro con otros sujetos que están inmersos en una misma situación, para intercambiar, corroborar, cuestionar, analizar y vivenciar experiencias vitales y emocionales compartidas. Y, por último, nos va a permitir crear un espacio de seguridad y de contención para avanzar en el proceso terapéutico que, en estas patologías graves, son largos y requieren estabilidad. Como la seguridad y la contención dependen de varias personas, y no de una sola, la continuidad de estos factores está, en cierta medida, garantizada. 
Consideraciones sobre la técnica grupal específica para el abordaje de pacientes esquizofrénicos

Teniendo en cuenta todo lo expuesto y considerando que el abordaje grupal del paciente esquizofrénico requiere de intervenciones que se adecuen a sus características concretas, vamos a intentar exponer algunas premisas que nos parecen necesarias en el tratamiento grupal de estos pacientes. En primer lugar, tenemos que tener en cuenta que trabajamos con pacientes que presentan graves alteraciones del lenguaje, del pensamiento, de la conducta y de la afectividad. Que tiene dificultades para la expresión y la comprensión verbal, presentan contenidos delirantes con interpretaciones distorsionadas de la realidad, presentan una conducta desorganizada acorde con sus percepciones delirantes y tienen dificultades para expresar sus afectos o lo hace de forma inapropiada. Tienen los límites de su identidad difusos y confunden lo que le es propio con los que es externo a ellos. En segundo lugar, hay que tener en cuenta que muchas emociones e, incluso, ideas, pueden expresarse mediante manifestaciones procedimentales.

Estas dos premisas que hemos planteado nos llevan a considerar las intervenciones técnicas más adecuadas en este tipo de grupos. En primer lugar, las intervenciones verbales tienen que ser expresadas en un lenguaje que, en todo momento, sea comprensible para los pacientes. En segundo lugar, los contenidos de las intervenciones verbales deben estar encaminados a centrar a los pacientes en la realidad, mediante el análisis y la comprensión dinámica de las distorsiones que hacer sobre ella, intentado que las intervenciones sean aclaratorias. En tercer lugar, hay que trabajar los contenidos del pensamiento, intentando la compresión de los contenidos delirantes. Es decir, intentando dar sentido a los contenidos delirantes de cada individuo. En cuarto lugar, la actitud del terapeuta debe ser cercana y afectiva, con los límites de la relación terapéutica claros y bien definidos. En quinto lugar, el rol de los terapeutas debe ser un rol activo, de soporte y contención de las ansiedades psicóticas (confusionales, paranoides, depresivas), durante todo el proceso de la terapia, aunque sabemos bien que el grupo mismo es el que acaba asumiendo esta tarea de contención. Y, por último, en el "aquí y ahora" del grupo analizaremos la forma de estar los pacientes con el resto de los miembros del grupo, señalando el tipo de relaciones que establecen con los demás y las conductas que generan en los otros. Lógicamente, habrá que señalar las dificultades y las características de los contactos afectivos, que suelen ser inapropiados, ausentes, etc.

La tarea grupal es, por tanto, la compresión de los pensamientos, acciones y sentimientos que los pacientes tienen sobre sí mismos y sobre el entorno, en el espacio grupal. Encaminado todo ello a promover el cambio en cada una de estas áreas, adecuándolas a la realidad, de forma que les permita una interacción más adaptada al entorno y una valoración más real de sí mismos. 


\section{BIBLIOGRAFÍA:}

(1) Garcia Badaracco, J. Psicoanálisis multifamiliar. Los otros en nosotros y el descubrimiento del sí mismo. Buenos Aires: Paidós, 2000.

(2) Méndez, J.A. e Ingelmo, J. La psicoterapia psicoanalítica desde la perspectiva del enfoque modular-transformacional. Introducción a la obra de Hugo Bleichmar. Madrid: Sociedad Forum de Psicoterapia Psicoanalítica, 2009.

(3) Bleichmar, H. Avances en psicoterapia psicoanalítica. Hacia una técnica de intervenciones específicas. Barcelona: Paidós, 1997.

(4) Freud, S. Esquema del psicoanálisis, 1940, Obras Completas, Vol. XXIII. Buenos Aires, Amorrortu, 1979, 133-209.

(5) Fromm-Reichmann, F. Principles of Intensive Psychotherapy. Chicago: Chicago Univ. Press, 1950.

(6) Fromm-Reichmann, F. Psychoanalysis and Psychotherapy. Chicago: Chicago Univ. Press, 1959.

(7) Searles, H.F. Collected Papers on Schizophrenia and Related Subjects. Nueva York: Int. Univ. Press, 1965.

(8) Ferenczi, S. Sin simpatía no hay curación. El diario clínico de 1932. Buenos Aires, Amorrortu, 1997.

(9) Federn, P. Ego Psychology and the Psychoses. Nueva York: Basic Books, 1952.

(10) Killingmo, B. Conflict and deficit: Implications for technique. Inter. J. Psycho-Anal., 1989; 70: 503-517.

(11) Killingmo, B. Affirmation in psychoanalysis. Inter. J. Psycho-Anal., 1995; 76: 65-79.

(12) Freud, S. Formulaciones sobre los dos principios del acaecer psíquico. 1911. Obras Completas, Vol. X II. Buenos Aires: Amorrortu, 1979; p. 219-223.

(13) Steiner, J. Psychic Retreats. Londres: Routledge, 1995.

(14) Freud, A. El yo y los mecanismos de defensa. 1936. Buenos Aires: Paidós, 1954.

(15) Klein, M. Notes on some schizoid mechanisms, 1946. The Writings of Melanie Klein, vol.3. Londres: Hogarth Press, 1986; p. 1-24.

(16) Freud, S. Un recuerdo infantil de Leonardo da Vinci. 1910. Obras Completas, Vol. XI, Buenos Aires, Amorrortu, 1979.p. 53-128.

(17) Aulagnier, P. La violence de l'interprétation. Du pictogramme a l'énoncé. Paris: Presses Universitaires de France, 1975.

(18) Ogden, Th. La frontera primaria de la humana experiencia. Madrid: Julián Yébenes, 1989.

(19) Bion. W. Learning from Experience. Londres: Heinemann, 1962.

(20) Rosenfeld, H. Impasse and Interpretation. Nueva York: Routlege, 1987.

(21) Segal, H. Algunos aspectos del análisis de un esquizofrénico, 1950. En H. Segal, La obra de Hanna Segal. Buenos Aires, Paidós, 1989; p. 137-161.

(22) Rayner, E. The Independent Mind in Brithish Psychoanalysis. Londres: Jasón Aronson, 1991.

(23) Winnicott, D. Los procesos de maduración y el ambiente facilitador. 1965. Barcelona: Paidós, 1993. 
(24) Balint, M. The Basic Fault: Therapeutic Aspects of Regresión. Londres:Tavistock, 1968.

(25) Kohut, H. The Restoration of the Self. Nueva York: Inter.Univ. Press, 1977.

(26) Kernberg, O. Borderline Conditions and Pathological Narcissism. Nueva York: Jason Aronson, 1975.

(27) Bion, W. A theory of thinking. En W. Bion, Second Thoughts. Londres: Heinemann, 1962; p. $110-119$.

(28) Stern, D., Sander, L., Nahum, J., Harrissson, A., Lyons-Ruth, K.,Morgan,A., et al. NonInterpretive Mechanisms in Psychoanalytic Therapy. The "something more" than interpretation. Int. J. Psycho-Analysis.1988, 79 (3): 903-921.

(29) Lyons-Ruth, M. El inconsciente bipersonal: el diálogo intersubjetivo, la representación relacional actuada y la emergencia de nuevas formas de organización relacional.Aperturas Psicoanalíticas, 2000. http://www.aperturas.org 